\section{Astrobiology at ten}

\section{A young discipline holds promise yet.}

n the 1960s, the evolutionary theorist George Gaylord Simpson rubbished the then-nascent science of exobiology, which concerned itself with life on places other than Earth, as a science without a subject.

Now the science descended from exobiology, astrobiology, is heading towards its tenth birthday. But as hundreds of its practitioners assemble this week in Washington for their annual AbSciCon meeting, the same criticism is being heard once more. And simpsonian scepticism that astrobiology has any useful data or insights to offer makes it easy to cut budgets for the discipline, as NASA has done (see Nature 439, 768-769; 2006). As we report on page 586 of this issue, these cuts have now been partially rescinded, but the discipline's future remains cloudy.

Setting aside for now the difficulties of allocating a constrained NASA science budget, the fundamental scepticism voiced by some of astrobiology's critics is misplaced, for at least two reasons. The first is the timescale of space science. Ten years is no time at all in terms of mission planning - and as a result there has not yet been a single astrobiological space mission. The first two that might make it off the launch pad will get under way in the next couple of years: the Kepler mission, which will look for planets the size of Earth circling other stars in orbits that might allow liquid water at the surface, and the Phoenix lander, which will look for organic molecules in ice on Mars. Both are important missions, Kepler profoundly so.

\section{Britannica attacks}

\section{.... and we respond.}

ast December, Nature published a News story about the accuracy of two online references sources. We compared the website of an established publication, Encyclopaedia Britannica, with that of Wikipedia, a new kind of online encydopaedia that anyone can edit and update, regardless of expertise.

The result (see Nature 438, 900-901; 2005) surprised us, and many others. Forty-two expert reviewers carried out the comparison. After we had tallied their results, we saw that they had picked up errors (the great majority of them minor) at a rate of about three per online Britannica item and about four per Wikipedia item.

Last week, Encyclopaedia Britannica issued a statement (http:// corporate.britannica.com/britannica_nature_response.pdf), and this week published a half-page advertisement in the London Times criticizing our study and demanding that we retract our story.

Britannica complains that we did not check the errors that our reviewers identified, and that some of them are not errors at all. We disagree with their claims in some of the cases (others are too specialized for an immediate response), but there is a more important point to make. Our reviewers may have made some mistakes - we have been open about our methodology and never claimed otherwise - but the entries they reviewed were blinded: they did not know which entry came from Wikipedia and which from Britannica. We see no reason to believe that any misidentifications of errors would adversely affect one publication more than the other. And of

The second reason is a misunderstanding - sometimes wilful, sometimes not - concerning the nature of astrobiology itself. Although the field was cooked up, in part, out of political necessity, as a means of bundling together research programmes on exobiology, other life sciences and planetary science, it has at its core a powerful unifying idea. Whereas exobiology specifically took life elsewhere in the Universe as the object of its study, astrobiology looks at life in the context of the Universe - in the context, that is, of astronomy and planetary science. Thus astrobiology legitimately broadens the terms of exobiology to include the study of life on Earth, which in this context is just another planet - albeit one to which we enjoy privileged access.

The inclusion of studies of life on Earth in astrobiology has provided opportunities to rebrand existing work. It appears that many microbiologists with an interest in extremophile microbes have suddenly become astrobiologists, because astrobiology is - or was -

"Ten yearsis no time in terms of mission planning - and there has not yet been a single astrobiology space mission."

where the money is. But it has also provided the field with a coherence that exobiology always lacked.

Life arises in an astronomical context, and Earth itself is part of that context. It is an intersection of the local and the cosmic, of the deepest time and the newest intelligence. Astrobiology has given this perspective an institutional home. Some second-rate research may have been funded on occasion, thanks to the astrobiology moniker's modishness. But the science indeed has a subject. It is a powerfully evocative one, which resonates not just with scientists, but with a wider public as well.

the 123 purported errors in question, Britannica takes issue with fewer than half.

Another Britannica criticism concerns the fact that we provided material from other Britannica publications, such as the Britannica Book of the Year. This was deliberate: the aim of our story, as we made clear, was to compare the online material available from Britannica and Wikipedia. When users search Britannica online, they get results from several Britannica publications. They have no reason to think that any one is less reliable than the others. In the case of some year-book entries, Britannica itself asks readers to reference the articles as coming from "Encyclopaedia Britannica Online" - exactly the

"Entries were blinded reviewers did not know which entry came from Wikipedia and which from Britannica."

source we set out to compare.

Other objections are simply incorrect. The company has, for example, daimed that in one case we sent a reviewer material that did not come from any Britannica publication. When the company made this point to us in private we asked for details, but it provided none. Now Britannica has identified the review in question as being on ethanol. We have checked the original e-mail that we sent to the reviewer who looked at the Britannica article on ethanol, and it is dear to us that all the reviewer's comments refer to specific paragraphs from Britannica.

Our responses to the points raised by Britannica in its original online posting and in its subsequent advertisement can be found at http://www.nature.com/nature/britannica/index.html. Our comparison was unbiased, and we reject Britannica's allegation that we have acted in a dishonest manner. We stand by the story. 